\title{
CHEMICAL INHOMOGENEITIES IN GALAXIES
}

\author{
M. G. Edmunds
}

Department of Applied Mathematics and Astronomy, University College, P.0. Box 78, Cardiff CFL 1XL.

The local chemical inhomogneity of the interstellar medium at a given time is an important factor in models of galactic chemical evolution. It can affect both the G-dwarf metallicity problem (Tinsley 1975, Talbot and Arnett 1973) and the correlation of the abundances of different elements (Tinsley 1976). Observational evidence of abundance gradients in our own Galaxy, and some other galaxies (summarised by Peimbert 1975, van den Bergh 1975), implies that in homogeneities over a large length scale must be created and survive during galactic evolution. Brief consideration of the mixing of the Galactic disk (e.g. Edmunds 1975, 1976) suggests that supernova remnant expansion, interstellar turbulence and differential-rotational shear are effective mixing mechanisms on a local scale.

Local Inhomogeneities Observational determination of the abundance of metals in stellar atmospheres has not yet reached the precision one might like, indeed an uncertainty of 0.2 in [Metals/H] is a fair rule of thumb for an individual analysis. Interpretation of abundance spread for a sample of stars is hindered by uncertainties in age determination and migration from place of birth in a radial abundance gradient. A reasonable representation for distribution of metal abundance $P(z)$ in stars of the same age, with mean $z^{\star}$, where $P(z) d z$ is the number of stars with metal abundance between $z$ and $z+d z$, is (Pagel and Pachett, 1975)

$$
P(z)=\frac{1}{2 \pi \sigma} \exp \left\{-\left(\log z-\log z^{\star}\right)^{2} / 2 \sigma^{2}\right\} .
$$

The typical difference $\delta z$ in metal abundance between two points in the disk at the time of star formation required to give the dispersion $\sigma$ may be shown to be of order $\delta z \sim 3 \sigma z^{*}$. Mayor (1976) and Pagel (1977, private communication) find a surprisingly low intrinsic spread in the abundance of young stellar groups near the sun, Mayor suggesting 
$\sigma_{[\mathrm{M} / \mathrm{H}]}=0.10\left(\begin{array}{c}+0.05 \\ -0.10\end{array}\right)$, consistent even with no variation. Such a small

spread may be telling us something about star formation in view of the list given in the following section of possible mechanisms which might induce inhomogeneity during star formation.

Freeman and Rodgers (1975) found a variation of composition of $R-R$ Lyrae stars in the globular cluster $w$ Cen, and suggested the observed stars had been formed after an initial generation had inhomogeneously enriched the proto-cluster material. It may be possible, however, to account for the anomalous line strengths by the effect on atmospheric composition and structure by the mixing up of material processed in the stellar interiors (Norris and Bessell, 1975, Besse11 and Norris, 1976) and retain initial local homogeneity.

Mechanisms to Produce the Unobserved Local Inhomogeneities

(i) Sequential star formation in associations, the first generation of supernovae enriching the material out of which further stars immediately form. There is considerable evidence that supernova explosions may induce further star formation. Sancisi (1974), Knapp and Kerr (1974) found shells of cold HI, dust and molecules around supernova remnants; Berkhuijsen (1974), Herbst and Assoussa (1977) found young stellar associations on the edge of supernova remnants. It has even been suggested (Cameron and Truran 1977) that our own solar system formation was induced by a supernova.

Even if the star formation is not actually triggered by supernova explosion, the synthesised metals from a supernova could still be incorporated, provided the formation happens soon afterwards (e.g. instigated by photoionized HII region formation, Elmegreen and Lada 1977) before the inhomogeneity is mixed out. If the products of several supernovae mixed into the mass of a typical observed she11 $\left(\sim 3 \times 10^{3} \mathrm{M}_{\Theta}\right)$, then an excess over surroundings of $\delta z \sim 3 \times 10^{-3}$ would be possible, resulting in $\sigma \sim 0.1$ for present mean disk abundances. Ogelmann and Maran (1976) suggest a repetative cycle of star formation $\vec{t}$ supernovae may operate within $O B$ associations. But the extent of inhomogneity will depend on how well and quickly the supernovae mix into the interstellar medium before new star formation takes place. The expanding remant will probably break up into knots (Chevalier 1975, 1976; Gul1, 1974) which may be metal rich (e.g. Cas A). It is not clear whether any induced star formation occurs from these knots before they mix into other material, or whether any stars formed come only by the implosion by the supernova shocking of previously existing, cool, normal abundance clouds. The existence of isotopic anomalies in meteorites might give some observational information, if interpreted as the contribution of pre-solar 
material (e.g. Arnould and Norgaard, 1977).

(ii) Since most of the heavy elements in the interstellar medium are in solid form (i.e. the "depletion" of elements onto grains, e.g. Morton et al 1973), fractionation between gas and grains during star formation could give rise to inhomogeneity both in the interstellar medium and in the forming stars. The effects of radiation pressure were considered by Edmunds and Wickramasinghe $(1974,1976)$, Yorke and Krugel (1976); accretion of cometary material by Joss (1974); gravitational segregation by Krautschneider (1977). Perhaps the smallness of the observed spread in abundance indicates that such processes do not operate, or that convective and turbulent mixing is more important.

(iii) Trapping of elements by magnetic fields by the Parker instability seems unlikely, since the random fluctuation of the Galactic magnetic field appears to be comparable to any systematic component (Wilkinson and Smith, 1974; Jokipii and Parker 1969).

\section{Large Scale Inhomogeneity}

In the disk of a spiral galaxy there is no shear mixing in the radial direction, and interstellar turbulence mixing times from $10 \mathrm{kpc}$ to $2 \mathrm{kpc}$ would typically exceed the Hubble time. Thus it is not unreasonable to suppose a radial abundance gradient could persist, although a given annular ring of say $200 \mathrm{pc}$ width in the disk should be well mixed. However, systematic radial gas flows may be important. The passage of a spiral shock can cause the gas to lose angular momentum, and hence fall slowly towards the centre. A simple model based on Roberts' (1972) estimate of non-closure of streamlines by $4 \%$ at the Sun's radius, and assuming angular momentum loss proportional to ( $\left.V_{\text {gas }}-V_{\text {pattern }}\right)$, with a suitably fitted Brandt rotation curve, implies that gas initially at the solar radius could fall to $2 \mathrm{kpc}$ in times of order $5 \times 10^{9}$ years. This seems sufficiently long to allow the persistence of a gradient, and anyway this is a one-way inflow and not a true homogenising process.

A study of HII regions across the Small Magellanic Cloud (Pagel et a1 1977) suggests a remarkably uniform composition, which could well be explained by good mixing due to streaming or circulating mass motions. Such motions would be expected if the SMC is really a barred system (de Vaucouleurs and Freeman, 1972). Since radial motions seem inevitable where a bar forms, other barred systems may be expected to be chemically uniform. An argument for inhomogeneity of the Large Magellanic Cloud has been made from the low abundance derived for the not-very-old outlying cluster NGC 2209 (Gascoigne et a1 1976), but a reconsideration of the analysis (Gustafsson et al 1977) suggests that its composition 
is in fact the same as that derived for HII regions.

It is interesting that the normal Sc galaxies $\mathrm{M} 33$ and $\mathrm{M} 101$ show abundance gradients while M51 does not (Compte 1975). This could well be due to the mixing effect of non-circular gas motions in M51 (Burbidge and Burbidge, 1964), probably resulting from perturbation by its close companion NGC 5195 .

Consideration of the formation of inhomogeneities during the initial rapid collapse phase of galaxies (e.g. Kaufmann 1975), both on a local and large scale, deserves further attention particularly in view of the elegant collapse models of Lynden-Be11 (1975) which neatly solve the G-dwarf problem. But for any such work a good theory of star formation is required.

\section{Conclusion}

Evolved galaxies appear to be remarkably well mixed, and probably the only chemical inhomogeneities of importance are large-scale radial gradients in galaxies lacking strong noncircular motions.

\section{REFERENCES}

Arnould, M., and Norgaard, H. 1977, preprint. Berkhuijsen, E.M. 1974, Atron. Astrophys . 35, 429. Besse11, M.S. and Norris, J. 1976, Ap. J. 208, 369.

Burbidge, E.M. and Burbidge, G.R. 1959, Ap. J., 140, 1445. Cameron, A.G.W. and Truran, J.W. 1977, Icarus, 30, 447. Chevalier, R.A. 1975, Ap. J. 200, 698. Chevalier, R.A. 1976 , Ap. J. $\overline{208}, 826$. Compte, G. 1975, Astron. Astrophys. 39, 197. Edmunds, M.G. 1975, Astrophys. Space Sci. 32, 483. Edmunds, M.G. 1976, Thes is, University of Cambridge. Edmunds, M.G. and Wickramasinghe, N.C. 1974, Astrophys. Sp. Sci. 30, L9. Edmunds, M.G. and Wickramasinghe, N.C. 1976, in Solid State Astrophysics, ed. N.C. Wickramas inghe and D.J.Morgan (Dordrecht, Reide1), p.233.

E1megreen, B.G. and Lada, C.J. 1977, Ap. J. 214, 725 . Freeman, K.C. and Rodgers, A.W. 1975, Ap. J. Lett. 201, L71. Gascoigne, S.C.B., Norris, J., Besse1, M.S., Hyland, A.R. and Visvanathan, N. 1976, Ap. J. Lett, $209,25$.

Gull, S.F. 1974, Mon. Not. R. Astr. Soc. $17 \overline{1}, 263$. Gustafsson, B., Be11, R.A. and Hejlesen, $\overrightarrow{\text { P.M. }}$ 1977, Ap. J. Lett. (in press).

Herbst, W. and Assoussa, G.E. 1977, preprint. Jokipii, J.R. and Parker, E.N. 1969, Ap. J. 155, 799. Joss, P.C. 1974, Ap. J. 191, 771. 
Kaufman, M. 1975, Astrophys. Space Sci. 33, 265.

Knapp, G.R. and Kerr, F.J. 1974, Astron. Astrophys. 33, 463.

Krautschneider, M.J. 1977, Astron. Astrophys. 57, 291.

Lynden-Be11, D. 1975, Vistas in Astronomy, 19, 299.

Mayor, M. 1976, Astron. Astrophys. 48, 301.

Morton, D.C., Drake, J.F., Jenkins, E.B., Rogerson, J.B., Spitzer, L. and York, D.G. 1973, Ap. J. Lett. 181, I103.

Norris, J. and Besse11, M.S. 1975, Ap. J. Lett, 201, L75.

Ogelmann, H.B. and Maran, S.P. 1976, Ap. J. 209, 124 .

Page 1, B.E.J., Edmunds, M.G., Fosbury, R.A.E. and Webster, B.L. 1977, in preparation.

Page1, B.E.J. and Patchett, B.E. 1975, Mon. Not. R. Astr. Soc. $172,13$.

Peimbert, M. 1975, Ann. Rev. Astron. Astrophys. 13, 113.

Roberts, W.W. 1972, Ap. J. 173, 259.

Sancisi, R. 1974 in Galactic Radio Astronomy, ed. F. J. Kerr and S. C. Simonson III (Dordrecht: Reide1), p.115.

Talbot, R.J. and Arnett, W.D. 1973, Ap. J. 186, 69.

Tinsley, B.M. 1975, Ap. J. 197, 159.

Tinsley, B.M. 1976, Ap. J. $208,797$.

Van den Bergh, S. 1975, Ann. Rev. Astron. Astrophys, 13, 217

Vaucouleurs, G. de., and Freeman, K.C. 1972, Vistas in Astronomy, 14,163 .

Wilkingson, A. and Smith, F.G. 1974, Mon. Not. R. astr. Soc. $167,593$.

Yorke, H.W. and Krugel, E. 1977, Astron. Astrophys. 54, 183. 